\title{
The effects of total and subtotal prefrontal cortex lesions on the timing ability of the rat
}

\author{
ARNE DIETRICH \\ Emory University School of Medicine, Atlanta, Georgia \\ DAVID L. FREDERICK \\ National Center for Toxicological Research, Jefferson, Arkansas \\ and \\ JOSEPH D. ALLEN \\ University of Georgia, Athens, Georgia
}

\begin{abstract}
Two peak procedure experiments studied the effects of different prefrontal cortex lesions on temporal discrimination in rats. In Experiment 1, lesions were made to either the medial frontal cortex (including medial precentral, anterior cingulate, prelimbic, and infralimbic cortex) or the ventral frontal cortex (including orbital area and agranular insular area) to determine the contribution of each to time estimation. In Experiment 2, lesions were made to the entire prefrontal cortex (including the medial frontal cortex and the ventral frontal cortex). In both experiments, rats were trained with a 40-sec peak interval and tested for 14 postoperative sessions. In Experiment 1, peak time and response distributions showed timing behavior unaffected by either lesion. In Experiment 2, ablations of the prefrontal cortex (PFC) produced a leftward shift in the temporal discrimination functions, indicating that these animals expected the time of reinforcement to be early. Also, these discrimination functions were substantially flattened, which was interpreted as a general decline in timing ability. These results are inconsistent with those of earlier studies. It is concluded that PFC functions as a unit with respect to timing behavior.
\end{abstract}

In recent years, researchers have started to investigate the underlying brain structures involved in the mechanism of timing. Studies have focused particularly on the prefrontal cortex (PFC; e.g., Meck, Church, Wenk, \& Olton, 1987; Olton, 1989; Olton, Wenk, Church, \& Meck, 1988). It has been suggested that the principle function of the PFC is the temporal integration of behavior (Fuster, 1980, 1989 ; Kolb, 1984). According to this hypothesis, the PFC organizes behavior across time and provides a basis for a timing mechanism.

For the timing of short duration, the model of the internal clock has provided a useful framework in which much of the data on timing behavior can be summarized (Church, 1978, 1984; Roberts, 1983; Roberts \& Church, 1978). Many of the details of this model have been gathered from data with the peak procedure. This procedure is based on a discrete fixed-interval (FI) schedule in which the availability of reinforcement is governed by a fixed time $(\mathrm{Ca}-$ tania, 1970; Roberts, 1981). The first response after this time is reinforced. A well-trained animal on a FI schedule shows an accelerated pattern of responding throughout the interval. After training, the animal is exposed to a few randomly interspersed peak trials that are indistin-

Correspondence should be addressed to A. Dietrich, Brain Research Lab, Department of Neurology, Emory University School of Medicine, Atlanta, GA 30322 (e-mail: adietri@emory.edu). guishable from the FI trials until the scheduled time of reinforcement. At this point, however, peak trials provide no reinforcement and the discriminative stimulus remains on for a much longer time. During these peak trials, the animal produces a response distribution that peaks near the scheduled time of reinforcement. Two measures are taken during peak trials: peak time and peak rate. Peak time is that point into the interval where the response rate reaches a maximum. Peak rate is the response rate at the peak time.

In the rat, the PFC is divided into the medial frontal and the ventral frontal cortex and is reciprocally connected to numerous brain structures (Groenewegen, 1988; Kolb, 1984; Krettek \& Price, 1974; Leonard, 1969). The most important connections with respect to timing are to the basal forebrain cholinergic system (BFChS) and to the limbic system (Meck, Church, \& Olton, 1984; Meck et al., 1987; Olton, Meck, \& Church, 1987). Of the BFChS nuclei, the basal forebrain nucleus receives the principal afferent fibers of the PFC, and the nucleus basalis magnocellularis (NBM) projects most densely to the PFC and, in particular, to the medial frontal cortex (Saper, 1984; Sesack, Deutch, Roth, \& Bunney, 1989). The limbic system has long been implicated in temporal integration, and parts of it have also been shown to affect timing ability (e.g., Meck et al., 1987). It has been known that the PFC projects to the entorhinal and perirhinal cortices, which provide input to the hippocampus (Krettek \& Price, 1974). 
However, the direct efferent connection of the hippocampus to the PFC was found only recently (Jay, Glowinski, \& Thierry, 1991). As with the NBM, these fibers innervate exclusively the medial frontal cortex. Also, the septal area projects to the medial frontal cortex.

Meck et al. (1987) used the peak procedure to investigate the role that $\mathrm{BFChS}$ and certain limbic system structures play in timing behavior. At the time of the study, two distinct brain systems were thought to be involved in timing behavior: the BFChS and the septo-hippocampal system. Meck et al. (1987) lesioned the originating and terminating structures of either pathway. For the septohippocampal pathway, the medial septal area (MSA) and the fimbria fornix (FF) were lesioned. For the other pathway, lesions were placed in the NBM and in the medial frontal cortex. The results revealed that MSA and FF animals shifted their peak distribution to the left, indicating that these animals expected the time of reinforcement to be early. In contrast, NBM and medial frontal cortex animals shifted their peak time to the right, indicating that the animal expected reinforcement opportunity to be late. From these data, it was concluded that the septo-hippocampal system and the BFChS are involved in timing ability, but in complementary ways.

There are various lines of reasoning that might challenge these conclusions. The first comes from neuroanatomy. The study of Meck et al. (1987) rested on the anatomical assumption that there are two distinct pathways that appeared to be involved in higher cognitive functioning: the septo-hippocampal system and the NBMmedial frontal pathway. These two pathways were repeatedly shown to be functionally dissociable in various cognitive tasks, and it was reasoned that the same might be true for timing behavior as tested by the peak procedure. Recent neuroanatomical evidence suggests, however, that the proposed destination of these pathways was oversimplified. It has been shown that these two pathways are interconnected at various points and even project to the same areas. Of particular interest has been the discovery that the hippocampus innervates the PFC directly (Jay et al., 1991). The projection field of those fibers was found to be exclusively the medial frontal cortex, which coincides with the main projection field of the cholinergic fibers arising from the NBM. Lesions to medial frontal cortex tissue would destroy the respective cell bodies of the NBM and the septo-hippocampus system. Also, the septal area was shown to project to the medial frontal cortex. These fibers travel even in the same bundle as the NBM fibers that innervate the medial frontal cortex (Saper, 1984). Thus, on a neuroanatomical basis, there is no reason to group the medial frontal cortex with the BFChS and no reason to consider it a distinct pathway with respect to the septo-hippocampal system.

This argument is supported further by evidence that lesions to the hippocampus proper failed to produce any impairment in timing ability with the peak procedure (Dietrich, Allen, \& Bunnell, in press); whereas, lesions to the FF do impair temporal memory (Meck, 1988; Meck et al., 1984; Olton et al., 1987). This indicates that dif- ferent parts of the septo-hippocampal system have different functions with respect to timing behavior, and it might not operate as a single temporal system at all. This further questions the assumption that two distinct systems operate to control timing ability and, in particular, that lesions to the medial frontal cortex affect any one timing system. It appears that the PFC possesses the appropriate input to perform integrative functions with respect to timing behavior.

A wealth of data over many decades has given support to the hypothesis that the PFC's primary function is the integration of behaviors across time (Fuster, 1980, 1989; Kolb, 1984). According to this hypothesis, there is no reason to suppose that lesions to the PFC would produce a disruption of the temporal discrimination function in any particular direction. Rather, the hypothesis predicts a more general disruption of the ability to time events. With the peak procedure, this would translate into a substantially leveled peak response distribution.

\section{EXPERIMENT 1}

The goal of Experiment 1 was to test whether a deficit in timing ability also appears in subtotal PFC lesions. Even though the experiment was not designed to test any neurotransmitter hypothesis, it originated from anatomical and pharmacological data that pointed at possibly different roles of neurotransmitter systems in the PFC with respect to timing ability.

Considerable evidence suggests that subjective estimation of event duration can be systematically altered through manipulation of neurotransmitter concentration (see Church, 1989). Specifically, drugs that affect dopamine (DA) and acetylcholine (ACh) produce the most dramatic and reliable changes in temporal memory. An increase above normal levels of DA and $\mathrm{ACh}$ results in overestimation of event duration, whereas decreases below normal levels cause underestimation (Maricq \& Church, 1983; Meck \& Church, 1987). Although producing similar results when altered, DA and ACh differ with respect to the onset and duration of their effects. Manipulations of DA levels produce temporary or phasic shifts in temporal discrimination (Maricq \& Church, 1983; Maricq, Roberts, \& Church, 1981; Meck, 1983, 1988), whereas treatments that affect $\mathrm{ACh}$ levels produce chronic shifts (Meck, 1983; Meck \& Church, 1987).

The rat PFC is subdivided into a medial frontal and a ventral frontal area (Kolb, 1984; Leonard, 1969). The medial frontal cortex receives significant innervation from the NBM, which is rich in ACh, but the ventral area receives substantial input from the striate cortex and rostal members of the A10 dopaminergic group (Beckstead, 1979; Gerfen \& Clavier, 1979; Leonard, 1972). If these areas were damaged selectively, one would predict that both would result in underestimation of time, thereby producing rightward shifts in peak time. However, the medial lesion, resulting in ACh deficits, would cause chronic shifts, whereas the ventral lesions, involving DA reduction, would produce only phasic shifts. 


\section{Method}

\section{Subjects}

Fifteen male Sprague-Dawley rats obtained from Sasco, Inc., 80 days old at the start of training were used in the experiment. Each rat was individually housed with free access to water but was deprived of food so that it remained at $85 \%$ of its free-feeding weight for the duration of the experiment. Supplementary daily rations of Purina Lab Chow were given after experimental sessions. A 12:12-h light:dark cycle, with fluorescent lights on at 6 a.m., was maintained at all stages of the experiment. Daily sessions were conducted between 1100 and $1600 \mathrm{~h}$.

\section{Apparatus}

Four identical Lehigh Valley Electronics (Model 1714) operant conditioning boxes $(28 \times 28 \times 24 \mathrm{~cm})$, each contained in its own sound-proof chamber, were used in the experiment. The boxes had standard response levers mounted on the left side of the control panel, $4 \mathrm{~cm}$ above the grid floor. A $5-\mathrm{V}$ dc cuelight located $5 \mathrm{~cm}$ above the lever was used as the discriminative stimulus. For appropriate lever responses, a standard formula $45-\mathrm{mg}$ Noyes food pellet was delivered as a reinforcer by a Davis Scientific Equipment (Model PD-190A) pellet dispenser into the central food cup. A Grason-Stadler (Model 901B) white-noise generator delivered $20 \mathrm{~dB}$ of white noise above ambient noise level.

All trials were controlled and recorded by four Commodore 8032 PET/CBM microcomputers, each interfaced with one operant chamber. All recorded data were later transferred to an IBM format for processing.

\section{Procedure}

Pretraining. All subjects were magazine and lever trained for 1-3 days until they responded continuously on a FI 1-sec schedule, whereby the first response occurring $1 \mathrm{sec}$ after the previous reinforced response produced a pellet.

Preoperative training. The subjects were exposed to daily 60-min peak procedure sessions for 70-120 days. For each session, approximately $70 \%$ of the trials were FI trials, and the remaining $30 \%$ were 90 -sec peak trials. The sequence of FI and peak trials was randomly determined by the microcomputer, and each was signaled by a white light that remained on for the duration of the trial. The light was turned off during a $10-\mathrm{sec}$ intertrial interval (ITI) that followed each trial. During FI 40-sec trials, a reinforcer was given for the first response $40 \mathrm{sec}$ after the onset of the light. During peak trials, the light remained on for $90 \mathrm{sec}$, and responses were recorded but were not reinforced. Responses were never reinforced during the ITI. Preoperative training was considered complete for each rat when it produced a stable response distribution.

Surgery. The subjects were randomly assigned to one of three groups: medial frontal, ventral frontal, or sham control. The rats were anesthetized by intraperitoneal (i.p.) injections of sodium pentobarbital and ketamine. The animals were fixed in a stereotaxic instrument, and the scalp was incised and retracted. At the appropriate site, small holes were drilled in the skull to perform the lesion. After the lesion, the animals were removed from the stereotaxic instrument, the skin was sutured, and then the animals were returned to their home cage.

For the medial frontal lesions, one opening was made at bregma, and another was made approximately $2 \mathrm{~mm}$ anterior to bregma. Rongeurs were used to extend the opening to $3 \mathrm{~mm}$ anterior to bregma and approximately $2.5 \mathrm{~mm}$ lateral to the central sinus. The medial frontal cortex underneath this opening was aspirated. For the ventral frontal lesions, an opening was made approximately $4 \mathrm{~mm}$ lateral of the sagittal suture beginning at bregma. The opening was then extended with rongeurs to $3 \mathrm{~mm}$ anterior to bregma and approximately $2.5-5 \mathrm{~mm}$ lateral to the central sinus. The ventral frontal cortex underneath this opening was aspirated. The sham animals were exposed to all steps of surgery except the actual aspiration of the cortex.
Postoperative testing. The subjects were permitted 7 days of recuperation following surgery, at which time they had free access to unlimited rat chow. Following 3 additional days that were required to reduce each subject's body weight to presurgery levels, 14 additional days of testing were conducted under the same contingencies that held during the training phase.

Histology. All animals were sacrificed with an overdose of $1 \mathrm{cc}$ sodium pentobarbital and then perfused through the heart with physiological saline and a $10 \%$ formalin and $90 \%$ saline solution. The brains were placed on a frozen-stage microtome, and the lesion site was sectioned coronally at $40 \mu \mathrm{m}$. Every fifth section was saved on a glass slide and stained with cresyl violet. Lashley grids were also used to verify the lesions.

Data collection and analysis. Data were collected separately for each subsection of an experimental session: the peak trials, the FI 40-sec trials, and the ITIs. Only data from the peak trials were processed further. This was done in the following manner: For each rat, the responses within individual peak trials were recorded in consecutive 1-sec bins. Each bin was summed over trials for each session and then divided by the number of peak trials to produce a mean response rate for that session. This mean response rate function was used to determine the peak time and peak rate of a session. A moving average based on consecutive 6 -sec epochs produced a smoothed response distribution. The middle of the interval that contained the maximum number of responses was defined as the peak time. On the instances where more than one interval contained the maximum number of responses, the peak time was defined as the median value between these two intervals. The peak rate was defined as the median response rate of the epoch, which made up the interval containing the peak time measure. This procedure was similar to the one used by Meck et al. (1987).

For each rat, a peak response distribution was obtained for the training phase and the testing phase. These distributions were based on the final 7 days of each phase. The individual distributions were then converted to percentage maximum response rate distributions in order to equalize the contribution of individual rats to the group mean peak time. An analysis of variance (ANOVA), with post hoc Tukey contrasts, was used to test for statistical significance.

\section{Results}

Figure 1 shows group mean peak times for the final 14 pre- and postsurgical sessions for the rats receiving control, medial, and ventral-frontal lesions. For all groups, peak times prior to surgery stabilized at values that were slightly higher than the nominal 40 -sec FI assigned by the schedule. An ANOVA revealed no difference among groups $[F(2,18)=0.3$, n.s.]. Postsurgery peak times for the control and ventral subjects did not appear to be systematically affected by the surgery. Peak times for 3 of the 5 subjects receiving medial lesions did drop dramatically during the first recovery session. However, for each subject, the drop was transitory, and peak time recovered by the second postsurgical session and remained at baseline for the remaining sessions. All Tukey comparisons showed no significant change in peak time from pre- to postoperative behavior for any group.

Peak rates over the final 14 pre- and postsurgery sessions for each group are also displayed in Figure 1. An ANOVA revealed a significant difference among groups $[F(2,18)=37.95, p<.0001]$. For the peak rate measure, an ANOVA also revealed a significant lesion $\times$ test condition interaction $[F(2,18)=33.83, p<.0001]$. For rats assigned to the control condition, peak rates varied from 35 to over 90 responses per minute, but Tukey compar- 


\section{Peak Time}
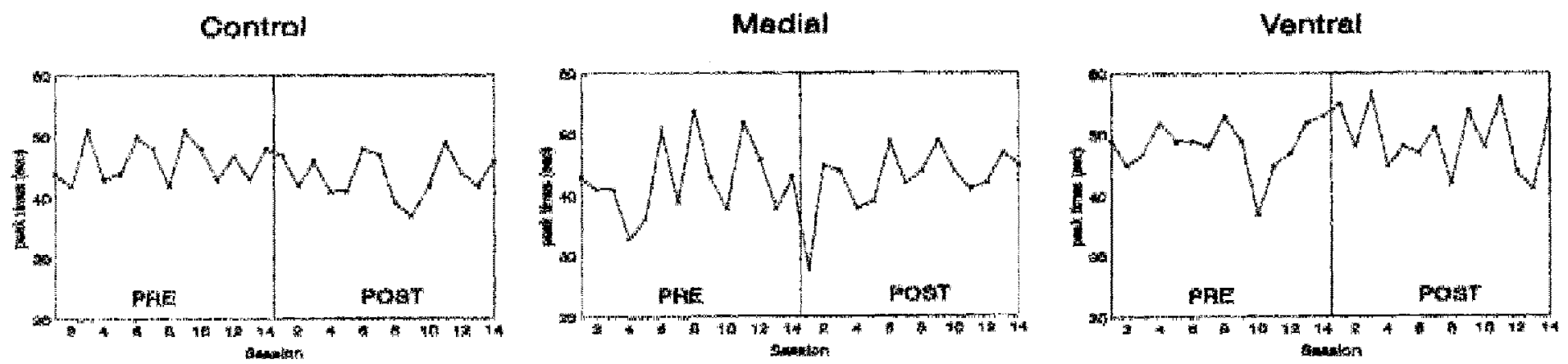

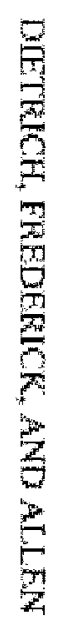

\section{Peak Rate}
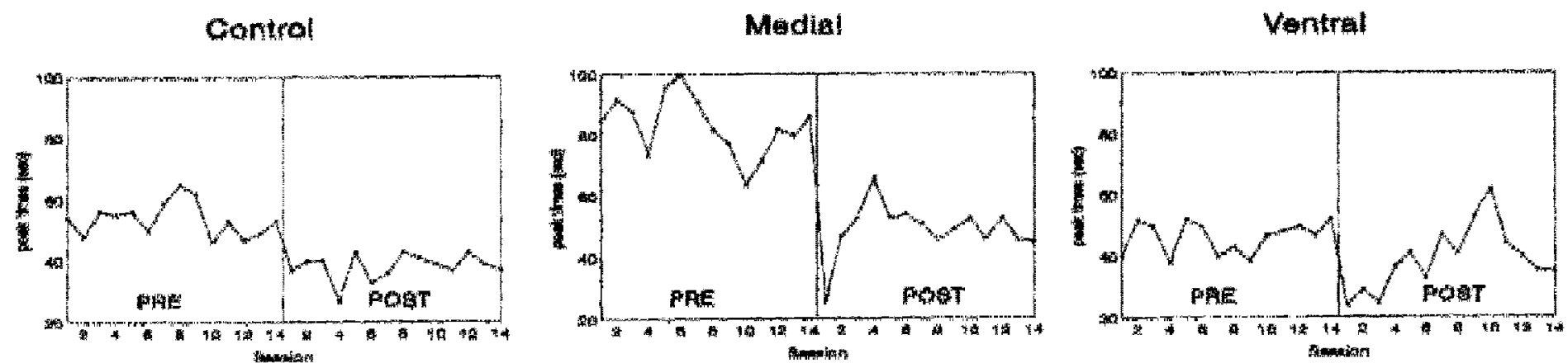

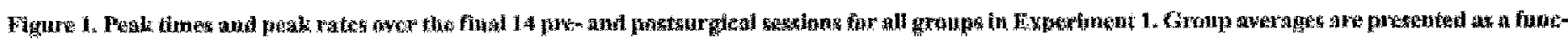
thon of gaspital. 
isons showed no change in the peak distributions from pre- to postoperative behavior for these rats. Only 1 subject showed a postsurgery drop that was dramatic and stable enough to lower the group's postoperative average slightly.

For rats receiving medial lesions, drops in peak rate were dramatic during the first postoperative session and remained depressed for 3 rats, but recovered quickly to baseline for the other 2 . The group mean record depicts an overall postoperative depression in peak rate, which is significant at the .05 level. Peak rate changes resulting from the ventral frontal lesion varied radically across subjects. As a result, the mean peak rate values for this group are not particularly representative.

Figure 2 depicts mean peak trial response rates from the final seven presurgery and postsurgery sessions as a function of the elapsed time into the peak trial for a representative subject from each group. For most subjects, the preoperative response rates over the 90 -sec peak interval roughly approximated a normal distribution, with its peak rate appearing slightly above the nominal 40 -sec reinforcement interval assigned by the FI 40 -sec training schedule. Comparing the pre- and postsurgery distributions of the control subjects, represented by $S-1$ in Figure 2, revealed only a further sharpening of the distribution, as would be expected if timing performance improved with further experience. There were no systematic shifts in peak between pre- and postoperative distributions for any rat. Response rate distributions for subjects in the medial and ventral groups, represented in Figure 2 by S- 9 and S-16, were similar to those in the control group, except that little sharpening of the postsurgery distribution occurred with any of the subjects. In fact, as can be seen in records for both S-9 and S-16, there was a small elevation in response rates at the tail of the postoperative distributions, which was characteristic of 4 of 5 subjects receiving medial lesions and 3 of 5 subjects given ventral frontal lesions.

Figure 3 shows the smallest and largest medial and ventral cortex lesions. The histology revealed no visible cortical damage for the rats in the sham control group. The subjects in either the medial or the ventral group had similar lesions in terms of surface area and depth. Lesions to the medial frontal cortex included the medial precentral, anterior cingulate, prelimbic, and infralimbic cortex. There was bilateral sparing of the infralimbic cortex in some rats and the ventral anterior cingulate cortex in others. Lesions to the ventral frontal cortex included the orbital area and the agranular insular area. There was no visible damage to the cingulate cortex or the forceps minor of the corpus callosum.

\section{Discussion}

Meck et al. (1987) imputed a slowing of the internal clocking mechanism to frontal cortex lesions, since their lesions produced a permanent rightward shift in the peak trial response distribution. A goal of Experiment 1 was to further refine the cortical areas involved in interval timing with more circumscribed lesions to the PFC. It was hypothesized that separate lesions to the ventral frontal and medial frontal areas would produce rightward shifts in the peak, similar to those reported by others (e.g., Meck et al., 1987; Olton, 1989), but that only the medial lesions would result in permanent shifts, the ventral frontal lesions producing phasic shifts. This experiment failed to demonstrate even convincing rightward phasic shifts in temporal distributions with either ventral or medial lesions, and, thus, little effect on timing ability in the rat was detected.

Although no significant changes in peak time were seen in Experiment 1, the overall ANOVA for the peak rate measure showed a significant difference among groups and a significant lesion $\times$ test condition interaction. Furthermore, postoperative peak rates were permanently lowered in 3 of 5 medial rats and were temporarily lowered in 2 rats in the ventral group. These peak rate changes represent the only consistent effect produced by either medial or ventral lesions, and their duration appeared to be consistent with the hypothesized lesion effects on peak times (i.e., chronic for medial subjects and phasic for ventral subjects). However, systematic changes in peak rate following frontal cortex lesions were not reported in the Meck et al. (1987) study.

The failure to find changes in peak time shows that neither lesion was sufficient to produce an impairment and suggests that the medial cortex and the ventral cortex are not singularly involved in timing behavior.

\section{EXPERIMENT 2}

Meck et al. (1987) reported a significant peak shift to the right for the frontal cortex group. This shift was chronic in nature and showed no signs of recovery throughout postoperative testing. It was concluded that the frontal cortex is functionally similar to the NBM but different from the septo-hippocampal system. As the results of Experiment 1 indicate, subtotal PFC lesions do not impair an animal's ability to tell time. A second experiment was therefore undertaken to study the effects of total PFC ablation on timing. This also served as part of a systematic replication of the research of Meck et al. (1987). It was hypothesized that total PFC lesions would result in a general decline of timing ability as indicated by a shift to the right and a substantially flattened peak distribution.

\section{Method}

\section{Subjects and Apparatus}

The experiment was performed on 4 male and 7 female LongEvans hooded rats, 90 days of age at the onset of testing, obtained from the University of Georgia Department of Psychology's breeding colony. Each rat was housed individually in a standard laboratory cage, given free access to water, and kept at $85 \%$ of its freefeeding body weight throughout the experiment. A daily ration of Purina Lab Chow was given to each rat shortly after the experimental session. A 12:12-h light:dark cycle, with fluorescent light on at 9 a.m., was maintained at all stages of the experiment. The apparatus was the same as in Experiment 1. 

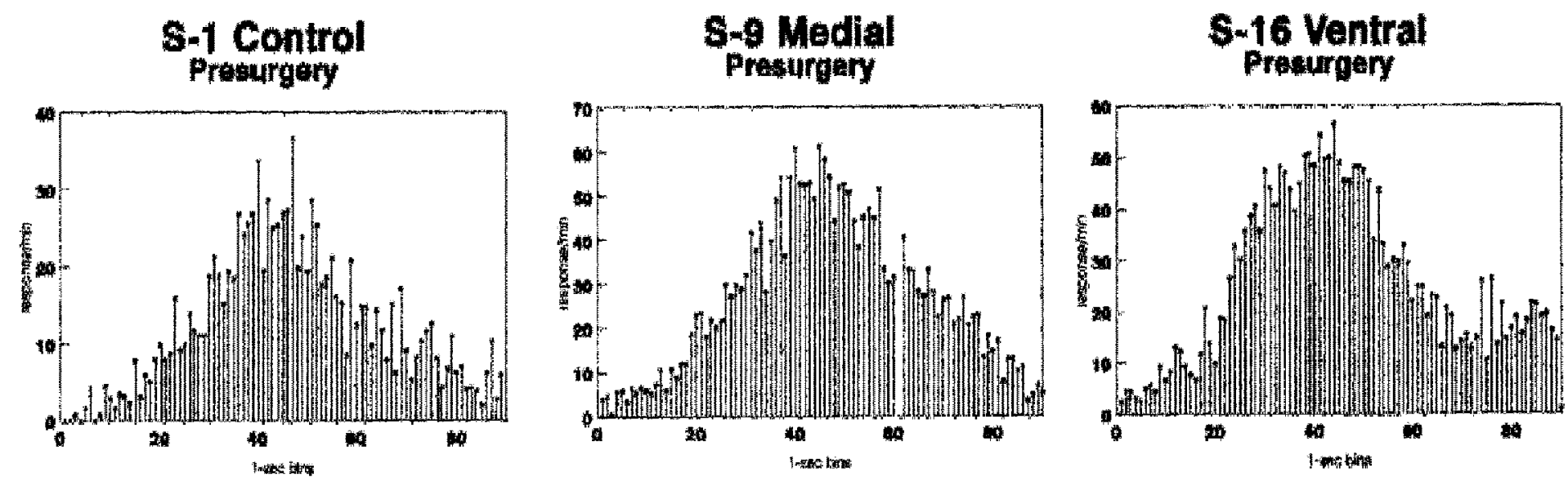

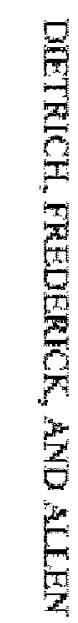
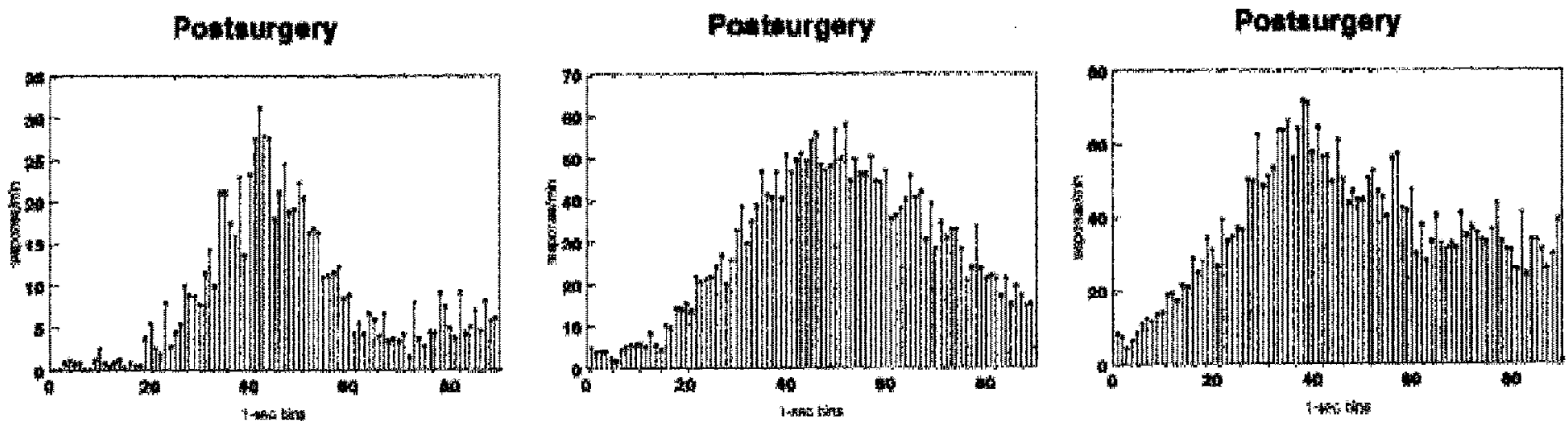

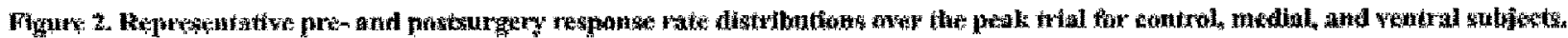




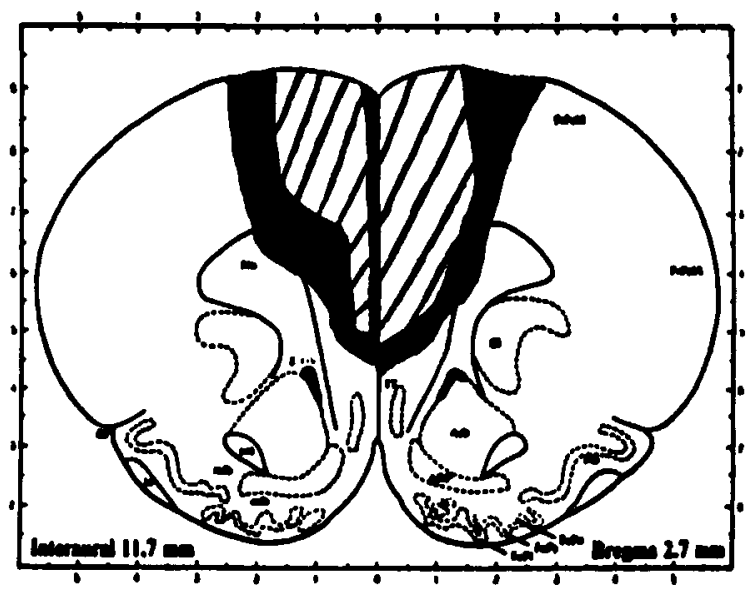

Small

Large

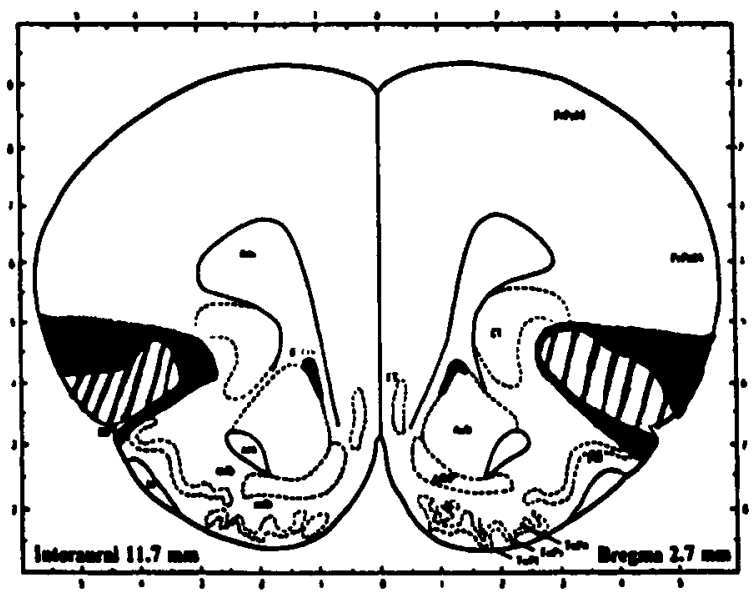

Figure 3. Representative coronal sections of the smallest and largest medial frontal (upper panel) and ventral frontal cortex (lower panel) lesions.

\section{Procedure}

Pretraining. Each rat was given 4 days of initial training, $1 \mathrm{~h}$ each day. For the first two sessions, food pellets were delivered on a fixed-time (FT) 60 -sec schedule, and each barpress also produced a reinforcer. In the next two sessions, the subjects received lever training until they showed reliable barpressing on a continuous reinforcement schedule.

Preoperative training. After response shaping, the rats were assigned randomly to three squads. Each squad was trained consistently for 120-min sessions at the same time of day. The first squad always started at 4 p.m., and the last squad finished at midnight. There were two types of trials, which began with the cuelight turned on. On FI trials, reinforcement was available after $40 \mathrm{sec}$. The first response after this FI produced food and ended the trial. On peak trials, food was never made available, and the discriminative stimulus remained on for $130 \mathrm{sec}$. Peak trials ended regardless of responding after $130 \mathrm{sec}$. FI trials constituted $65 \%$ of all trials, and peak trials constituted $35 \%$ of all trials. The trials were separated by an ITI of $60 \mathrm{sec}$, during which the discriminative stimulus was switched off and responding was ineffective. Depending on the subject, preoperative training required between 87 and 100 sessions to produce a stable response distribution. When a rat had reached stable baseline responding, it was subjected to surgery.

Surgery. The subjects were randomly assigned to either the surgery group or the sham control group in such a way that the experimental group contained twice as many subjects. The subjects were anesthetized with a mixture of i.p. injections of ketamine $(90 \mathrm{mg} / \mathrm{kg})$ and xylazine $(5 \mathrm{mg} / \mathrm{kg})$ and prepared for the lesion as described in Experiment 1.

The frontal cortex lesions were performed by aspiration with the aid of a Bausch \& Lomb Stereo Zoom 5 microscope. An opening in the skull was made that measured approximately 1-3 mm anterior to bregma and bilaterally $1-4 \mathrm{~mm}$ lateral to the central sinus. The frontal cortex underneath was aspirated. An effort was made to spare the fibers of the corpus callosum. The resulting cavity was stuffed with sterile gelatin foam.

Testing. After a 10-day recovery period following surgery and an additional 4 days of food deprivation, all animals resumed training. The testing phase was conducted under the same contingencies that held for the training phase. All animals were exposed to 14 testing sessions.

\section{Results}

\section{Baseline (Days ca. 87-100)}

During the last 7 days of baseline acquisition, all rats had developed a stable response distribution with a mean peak time of $41.3 \pm 6.1 \mathrm{sec}$ and a mean peak rate of $60.7 \pm 23.7$ responses per minute. An ANOVA followed by Tukey comparisons showed that peak time and peak rate measures did not differ among groups.

\section{Peak Trials (Days ca. 88-114)}

Figure 4 shows the last 7 sessions of baseline acquisition and all 14 postoperative sessions for both groups. Mean peak times and rates are presented as a function of session. For the peak time measure, an ANOVA revealed only a significant lesion $\times$ test condition interaction $[F(1,9)=10.66, p<.01]$. Tukey comparisons showed no change in the peak distributions from pre- to postoperative behavior for rats in the control group. For the PFC animals, Tukey comparisons demonstrated a significant shift to the left in the postoperative distributions, indicating that the internal clock signaled the reinforcement event earlier than it actually occurred. The overall development of this peak shift appeared to be chronic. The onset of the shift was late, and, over several sessions, the peak shifted slowly to earlier times in the peak interval. The animals never recovered their preoperative peak times, and the shift appeared permanent.

A number of other significant changes were also observed in PFC rats. Relative to the operated controls, PFC rats needed more testing sessions to relearn the peak procedure after surgery. During the first three postoperative sessions, some of these rats showed no timing ability at all. Their peak distributions were flat. Also, multiple-peak distributions were quite common. In contrast, after some initial relearning trials, control animals showed no impairment in estimating the time of reinforcement opportunity. Figure 5 shows individual data that compare preand postoperative performance for 2 representative sub- 
Mean peak times

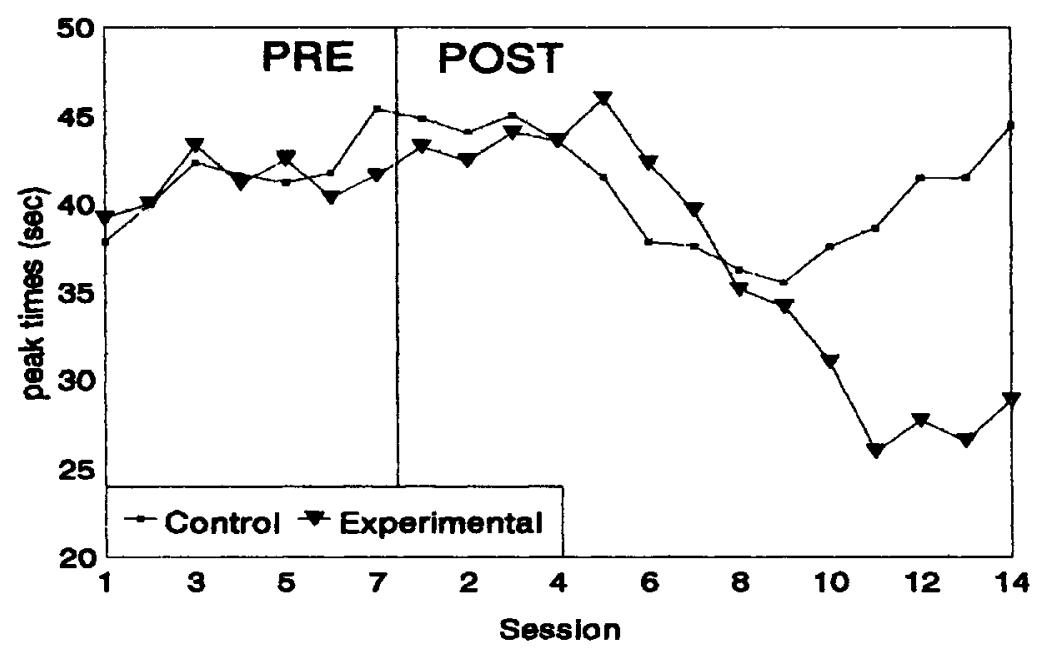

Mean peak rates

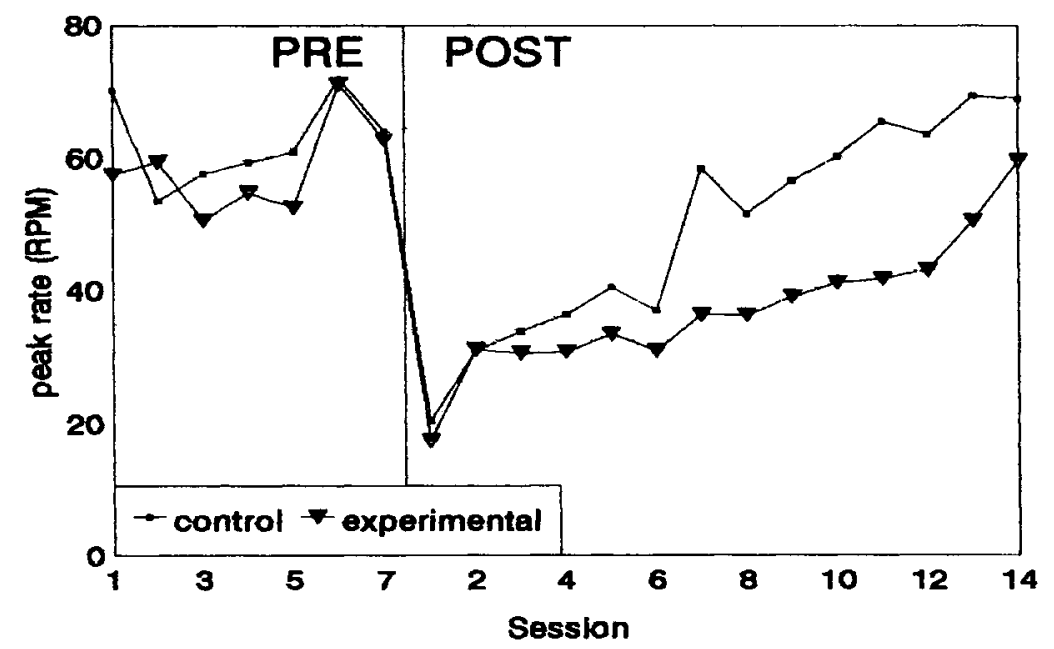

Figure 4. The last 7 days of baseline acquisition and all 14 postoperative sessions for both groups in Experiment 2. Mean peak time and mean peak rate are presented as a function of session.

jects. Relative to the controls, there is an overall flattening of the postoperative peak distribution for PFC rats. Furthermore, some PFC rats also displayed noticeable hyperactivity, which has been documented for medial frontal cortex lesions (Kolb, 1984).

For the peak rate measure, an ANOVA showed no significant main or interaction effects. Following surgery, however, both groups demonstrated a marked and immediate depression of peak rate. Slowly, but consistently, peak rate in both groups increased and reached levels that approximated preoperative baseline levels. The PFC group recuperated somewhat more slowly than the control group did, which may be attributed to the marked decline of 1 rat's peak rate. This subject's histology indicated some damage to the olfactory bulbs.

\section{Histology}

Figure 6 shows the smallest and largest PFC lesions. The sham operations for the rats in the control group resulted in no visible cortical or subcortical damage. Therefore, no further analysis was performed on their brains. All subjects in the PFC group had similar lesions in terms of surface area. Most of the PFC tissue was successfully removed and included the medial frontal cortex and the ventral frontal cortex. There was bilateral sparing of parts of the ventral anterior cingulate cortex, the lateral agranular insular area, and the infralimbic area in different subjects. Some subjects had minor damage extending medially into the cingulate cortex. No subject showed any damage that extended dorsally into the premotor area. Only 1 subject had minimal damage to the olfactory bulbs just rostal to the medial 


\section{Subject 26 Lesion}

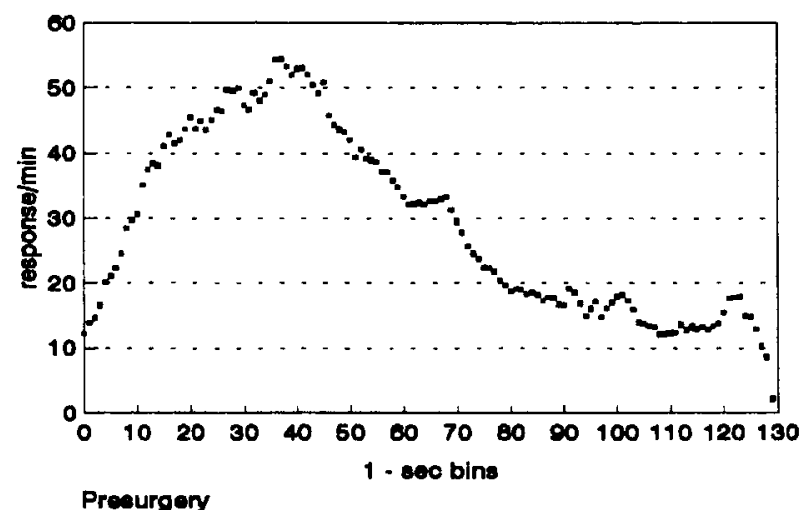

Preeurgery

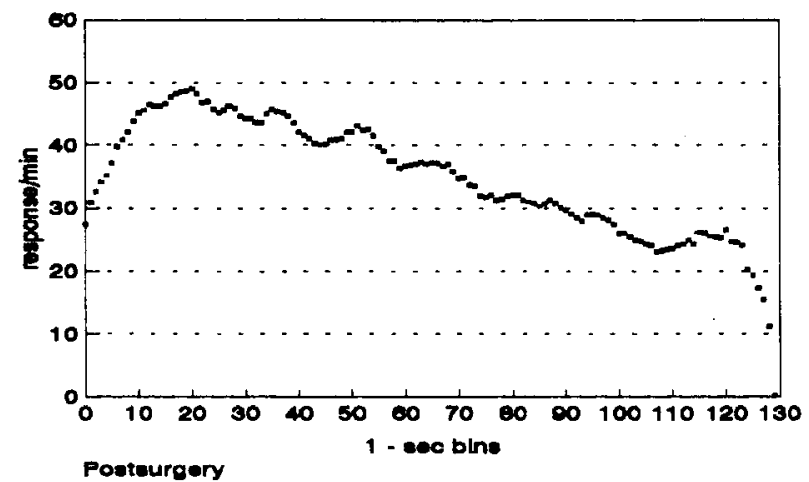

Subject 05 Lesion
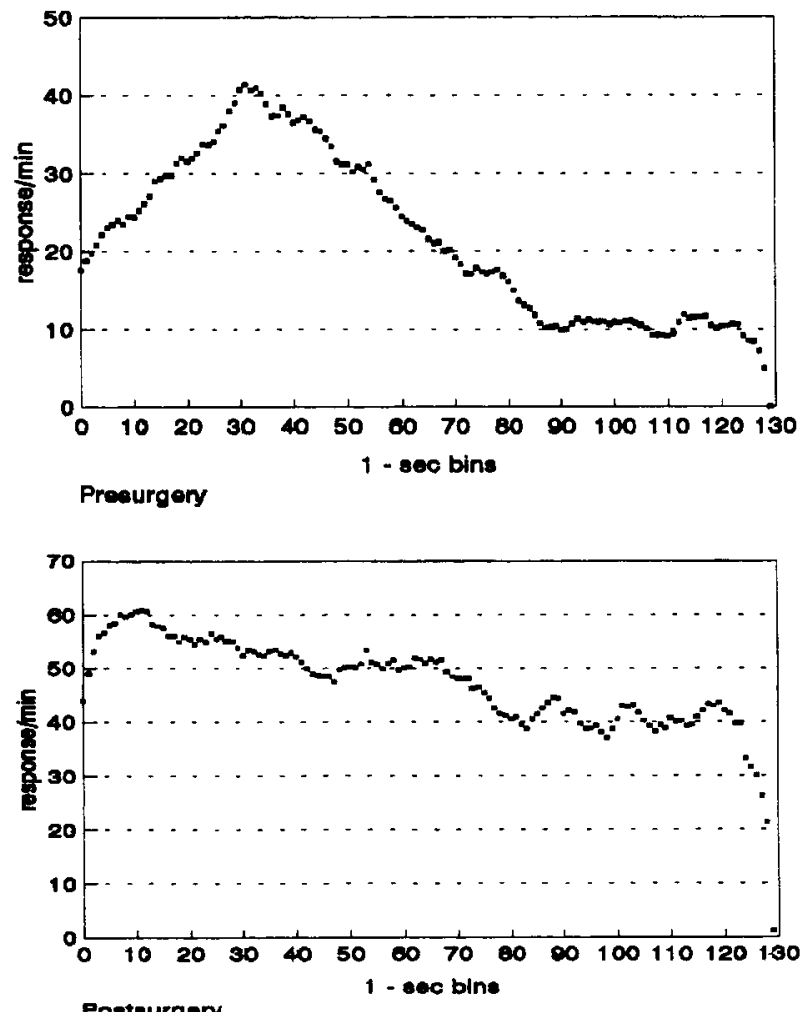

Figure 5. Pre- and postoperative performance for 2 representative subjects. PFC animals show a much flattened postoperative peak distribution.

frontal cortex. In terms of depth, the lesions were less uniform. In 4 subjects, the cortex was successfully aspirated, sparing the underlying fiber tracts of the corpus callosum. The histology of the remaining 3 subjects revealed some minor subcortical damage. This damage was unilateral and affected mainly the fiber tracts of the corpus callosum. In 2 subjects, the lesion extended into the corpus callosum on the left hemisphere; whereas, the other subject showed similar callosal damage on the right hemisphere.

\section{Discussion}

Experiment 2 demonstrated that total ablations of the PFC affect the timing ability of the rat. The ablations both flattened and shifted the temporal discrimination function to the left. The shift appeared to be irreversible and chronic in nature. This chronic leftward shift translates into a decrease in peak time, which indicates that the animal's internal clock signaled the event of reinforcement opportunity to be early. It was also observed that PFC lesions temporarily decreased the peak rate.

The purpose of Experiment 2 was to study the role played by the entire PFC in timing performance. It also served as part of a systematic replication of the results of Meck et al. (1987). Experiment 2 was only partially successful in replicating these results. Consistent with Meck et al. (1987), it was found that damage to the PFC causes chronic shifts; however, inconsistent with their findings, Experiment 2 revealed a peak shift to the left.

Furthermore, the results of this experiment give only partial support to the hypothesis that PFC lesions do not produce specific impairment in the ability to estimate time. Since the PFC appears to be involved in integrating the temporal aspects of behavior, it was hypothesized that only nonspecific deficits would occur. Consistent with this view was the observation of flattening of the postoperative timing distributions. The animals' peak response distributions were relatively flat throughout postsurgical testing, and they never approximated the Gaussian curve that was typical for the control subjects' or the lesioned animals' own preoperative distributions.

\section{GENERAL DISCUSSION}

In Experiment 1, it was demonstrated that smaller and more restricted PFC lesions have no effects on the temporal discrimination function. Neither ventral frontal nor medial frontal cortex lesions revealed impairments in the timing task. However, in Experiment 2, total lesions of the PFC resulted in a permanent alteration of the temporal discrimination function. Response distributions were 


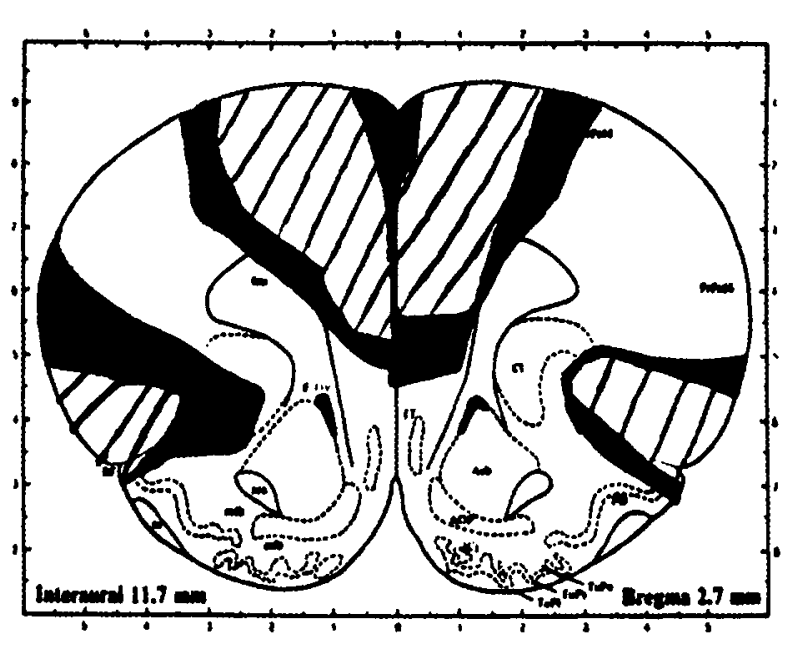

Small

Large

Figure 6. Representative coronal sections of the smallest and largest PFC lesions.

substantially flattened, and the peak time was chronically shifted to the left.

This set of results suggests that the amount of removed cortical tissue is directly related to the ability to estimate the passage of time. Neither the ventral frontal area nor the medial frontal area appears to be singularly involved in timing behavior, but both are necessary for accurate timing to occur. Therefore, it is argued here that the integrative function of the PFC is only possible when the PFC operates as a whole unit. Different parts of the PFC receive input from different structures and send fibers to another different set of structures. Thus, it is reasonable to assume that distinct parts of the PFC contribute differently to the temporal integration of behavior. The disruption of one part of the PFC can apparently be compensated for by the other. However, the disruption of the entire PFC cannot be compensated for by other cortical areas.

The PFC possesses ample afferent and efferent connections to all subcortical structures that have been shown to change the temporal discrimination function of the peak response distribution. It would be naive to assume that the damage in this study was limited to the PFC. Underlying fibers, the cell bodies of afferent input, the efferent projection areas, and passing bundles are affected by these lesions. Therefore, it is good to be cautious when inferring that any effect observed after ablation is due to the restricted area that was removed -in particular, when the dependent variable is a broad measure, such as time discrimination. It is not known how the hypothesized temporal integration takes place or which subcortical structure contributes to it and in what strength. It is conceivable that input from one subcortical structure has more impact on the ability to tell time than input from another.
If the PFC is removed, any specific impairment that is subsequently observed could still be attributed to the integration function rather than to a specific function of the PFC. One subcortical system may simply exercise more control over time estimation than another and, without the PFC, will not be checked before the behavioral outcome occurs. This possibility is advanced here as a plausible explanation for the specific impairment, a leftward shift of the temporal discrimination function.

Meck et al. (1987) hypothesized that the lesions of the frontal cortex impaired reference memory. The explanation given for this conclusion was the chronic shift in timing distribution that was observed in those subjects. Chronic shifts are taken as impairment of memory because of their latency of onset. If reference memory is damaged the animal detects the time in the accumulator as reliably different from the time in reference memory (see Church, 1989). After some time or trials, however, reference memory will store the constantly occurring new value and slowly adjust to it. This results in the chronic shift with ongoing treatment. If treatment is terminated, reference memory will again accommodate as the internal clock produces a reliably different time. Phasic shifts, on the other hand, are produced by changes in the clock itself. Starting from the first trial after treatment onset, the clock deviates from the reference point - that is, the remembered time of previous trials. Therefore, the clock runs until it matches this reference point, and peak time shifts are instantaneously obtained as a consequence. There is recovery with more experience, however. These are reasonable assumptions, and, consequently, it is concluded that the chronic shift pattern observed in Experiment 2 resulted from reference memory impairment.

The temporal structuring of events and behaviors is essential for the survival of the organism. Without the ability to time events relative to other events, an organism is unable to detect which event precedes another and is therefore not capable of making inferences about causal relationships between events. There would be no adaptation process and, subsequently, no survival. It is hard to imagine that an ability so essential for survival would be controlled by the PFC, which is phylogenetically very young. This is consistent with observations that circadian rhythms and seasonal fluctuations seem to be controlled minimally, if at all, by the PFC. It might be argued that every part of the brain has its own internal clock, in the sense that every structure that controls a given behavior also controls the temporal aspect of that behavior. This idea of timing ability would be somewhat similar to the picture that has emerged for memory functions. Like memory, the internal clock is not centralized, and, therefore, every part of the brain contributes something to the ability to tell time. The PFC may function to integrate this information. It is even conceivable that some temporal integration takes place at the limbic system level. The limbic system is hypothesized to play a crucial role in the execution of behavior at the appropriate time and place. One consequence of this picture of timing behavior is that 
most brain structures, if destroyed, would result in some impairment of time perception, time production, or both.

\section{REFERENCES}

BeCKSTEAd, R. M. (1979). An audioradiographic examination of corticocortical and subcortical projections of the mediodorsal-projection (prefrontal) cortex in the rat. Journal of Comparative Neurology, 184, 43-62.

Catania, A. C. (1970). Reinforcement schedules and psychophysical judgment: A study of some temporal properties of behavior. In W. N. Schoenfeld (Ed.), The theory of reinforcement schedules (pp. 1-42). New York: Appelton-Century-Croft.

Church, R. M. (1978). The internal clock. In S. H. Hulse, H. Fowler, \& W. K. Honig (Eds.), Cognitive processes in animal behavior (pp. 277-310). Hillsdale, NJ: Erlbaum.

CHURCH, R. M. (1984). Properties of the internal clock. In J. Gibbon \& L. Allan (Eds.), Timing and time perception (Annals of the New York Academy of Sciences, Vol. 423, pp. 566-582). New York: New York Academy of Sciences

Church, R. M. (1989). Theories of timing behavior. In S. B. Klein \& R. R. Mowrer (Eds.), Contemporary learning theories (pp. 47-71). Hillsdale, NJ: Erlbaum.

Dietrich, A., Allen, J. D., \& Bunnell, B. N. (in press). Is the hippocampus involved in temporal discrimination and the memory of short intervals? International Journal of Neuroscience.

FuSTER, J. M. (1980). The prefrontal cortex. New York: Raven.

FUSTER, J. M. (1989). The prefrontal cortex: Anatomy. physiology and neuropsychology of the frontal lobe (2nd ed.). New York: Raven.

Gerfen, D. R., \& Clavier, R. M. (1979). Neural inputs to the prefrontal agranular cortex in the rat: Horseradish peroxidase study. Brain Research Bulletin, 4, 347-353.

GROENEWEGEN, H. T. (1988). Organization of the afferent connections of the mediodorsal thalamic nucleus in the rat, related to the mediodorsalprefrontal topography. Neuroscience, 24, 379-431.

JAY, T. M., Glowinski, J., \& THIERRY, A. M. (1991). Selectivity of the hippocampal projection to the prelimbic area of the prefrontal cortex in the rat. Brain Research, 505, 337-340.

KoLB, B. (1984). Functions of the prefrontal cortex in the rat: A comparative view. Brain Research Review, 8, 65-98.

Krettek, J. E., \& Price, J. L. (1974). A direct input from the amygdala to the thalamus and the cerebral cortex. Brain Research, 67, 169-174.

LEONARD, C. M. (1969). The prefrontal cortex in the rat. I. Cortical projections of the mediodorsal nucleus. II. Efferent connections. Brain Research, 6, 321-343.

LEONARD, C. M. (1972). The connections of the dorsomedial nuclei. Brain. Behavior, \& Evolution, 6, 524-542.
Marice, A., \& Church, R. M. (1983). The differential effects of haloperidol and methamphetamine on time estimation in the rat. Psychopharmacology, 79, 10-15.

MARICQ, A., Roberts, S., \& Church, R. M. (1981). Methamphetamine and time estimation. Journal of Experimental Psychology: Animal Behavior Processes, 7, 18-30.

MECK, W. H. (1983). Selective adjustment of the speed of the internal clock and memory processes. Journal of Experimental Psychology: Animal Behavior Processes, 9, 171-201.

MECK, W. H. (1988). Hippocampal function is required for feedback control of internal clock's criterion. Behavioral Neuroscience, 102, 54-60.

Meck, W. H., \& ChurCh, R. M. (1987). Cholinergic modulation of the context of temporal memory. Behavioral Neuroscience, 101, 457-464.

Meck, W. H., Church, R. M., \& Olton, D. S. (1984). Hippocampus, time and memory. Behavioral Neuroscience, 98, 3-22.

Meck, W. H., Church, R. M., Wenk, G. L., \& Olton, D. S. (1987). Nucleus basalis magnocellularis and medial septal area lesions differentially impair temporal memory. Journal of Neuroscience, 7 , 3505-3511.

Olton, D. S. (1989). Frontal cortex, timing, and memory. Neuropsychologia, 27, 121-130.

Olton, D. S., Meck, W. H., \& ChurCh, R. M. (1987). Separation of hippocampal and amygdaloid involvement in temporal memory dysfunctions. Brain Research, 404, 180-188.

Olton. D. S., Wenk, G. L., Church, R. M., \& Meck, W. H. (1988). Attention and the frontal cortex as examined by simultaneous temporal processing. Neuropsychologia, 26, 307-318.

ROBERTS, S. (1981). Isolation of an internal clock. Journal of Experimental Psychology: Animal Behavior Processes, 7, 242-268.

Roberts, S. (1983). Properties and function of an internal clock. In R. L. Mellgren (Ed.), Animal cognition and behavior. Amsterdam: North-Holland.

Roberts, S., \& Church, R. M. (1978). Control of an internal clock. Journal of Experimental Psychology: Animal Behavior Processes, 4, 318-337.

SAPER, C. B. (1984). Organization of cerebral cortical afferent systems in the rat: II. Magnocellular basal nucleus. Journal of Comparative Neurology, 222, 313-342.

Sesack, S. R., Deurch, A. Y., Roth, R. H., \& Bunney, B. S. (1989). Topographical organization of efferent projections of the medial prefrontal cortex in the rat: An anterograde tract-tracing study with Phaseolus vulgaris leucoagglutinin. Journal of Comparative Neurology, 290, 213-242.

(Manuscript received July 18, 1996; revision accepted for publication December 23, 1996.) 